\title{
Recent Advances in Diagnosis and Management of Female Genital Tuberculosis
}

\author{
J. B. Sharma ${ }^{1}\left[\right.$ Eshani Sharma $^{1} \cdot$ Sangeeta Sharma ${ }^{2} \cdot$ Sona Dharmendra ${ }^{1}$
}

Received: 26 April 2021 / Accepted: 28 June 2021 / Published online: 28 August 2021

(c) Federation of Obstetric \& Gynecological Societies of India 2021

\begin{abstract}
Female genital tuberculosis (FGTB) is an important cause of significant morbidity and infertility.

Gold-standard diagnosis by demonstration of acid fast bacilli on microscopy or culture or detection of epithelioid granuloma on histopathology of endometrial or peritoneal biopsy is positive in only small percentage of cases due to its paucibacillary nature. Use of gene Xpert on endometrial or peritoneal biopsy has improved sensitivity of diagnosis. Composite reference standard (CRS) is a significant landmark in its diagnosis in which combination of factors like AFB on microscopy or culture, positive gene Xpert, epithelioid granuloma on endometrial or peritoneal biopsy, demonstration of definite or probable findings of FGTB on laparoscopy or hysteroscopy. There have been many advances and changes in management of FGTB recently. The program is now called National Tuberculosis Elimination Program (NTEP), and categorization of TB has been stopped. Now, patients are divided into drug-sensitive FGTB for which rifampicin $(\mathrm{R})$, isoniazid $(\mathrm{H})$, pyrazinamide $(\mathrm{Z})$ and ethambutol (E) are given orally daily for 2 months followed by three drugs (rifampicin, isoniazid and ethambutol (RHE) orally daily for next 4 months. Multi-drug-resistant FGTB is treated with shorter MDR TB regimen of 9-11 months or longer MDR TB regimen of 18-20 months with reserved drugs. In vitro fertilization and embryo transfer have good results for blocked tubes and receptive endometrium, while surrogacy or adoption is advised for severe grades of Asherman's syndrome.
\end{abstract}

Keywords Female genital tuberculosis · Drug-sensitive tuberculosis · Drug-resistant TB $\cdot$ Composite reference standard · Laparoscopy $\cdot$ Hysteroscopy

Dr. J. B. Sharma MD, DNB, FRCOG (London), PhD, MFFP FAMS, FICOG FIMSA, is a Professor in Department of Obstetrics and Gynecology, All India Institute of Medical Sciences, New Delhi; Eshani Sharma, Senior Research Fellow, MBBS in Department of Obstetrics and Gynecology, All India Institute of Medical Sciences, New Delhi; Sangeeta Sharma, Professor and Head, MD in Department of Paediatrics, National Institute of Tuberculosis and Respiratory Diseases, New Delhi; Sona Dharmendra, Ph. D Scholar, pursuing PhD in Department of Obstetrics and Gynecology, All India Institute of Medical Sciences, New Delhi.

J. B. Sharma

jbsharma2000@gmail.com

1 Department of Obstetrics and Gynaecology, All India Institute of Medical Sciences, Room No. 3064A, IIIrd Floor, Teaching Block, New Delhi 110029, India

2 Department of Paediatrics, National Institute of Tuberculosis and Respiratory Diseases, New Delhi, India

\section{Introduction}

Tuberculosis remains a major public health problem globally [1]. Female genital tuberculosis (FGTB) is a chronic infectious disease of female genital tract due to Mycobacterium tuberculous (rarely Mycobacterium bovis from unpasteurized infected milk) [2, 3]. It causes significant morbidity, especially irregular menstruation, pelvic pain, tubo-ovarian mass and infertility particularly in South East Asia and Africa [4-9]. The disease is making a comeback even in the developed countries like Europe and USA due to more liberal migration, concomitant immunocompromising diseases like human immunodeficiency virus coinfection, diabetes mellitus, chronic kidney and other systemic diseases [8-10]. FGTB is usually secondary to pulmonary TB through hematogenous route or lymphatic route but can occur through contagious spread from adjacent ileocaecal TB and rarely through infected semen from tuberculous orchitis in males [5, 6]. FGTB can cause permanent damage to female genital tract in advanced stages. Hence, early 
diagnosis and treatment in subclinical stage can prevent permanent damage and sequelae of female genital tract with successful pregnancy outcome $[11,12]$. A high index of suspicion is needed for diagnosis which includes past or family history of TB or anti-tubercular therapy [4]. There have been recent changes in diagnosis and treatment of all types of TB including FGTB $[13,14]$. Although FGTB is a paucibacillary disease, still all efforts should be made to arrive at a microbiological diagnosis and to do drug sensitivity test as treatment of TB including FGTB is now drug sensitivity test (DST) based using oral daily 4 drugs (rifampicin, isoniazid, pyrazinamide and ethambutol, RHZE for 2 months) followed by three drugs (RHE) daily orally for next 4 months. For drug-resistant TB, MDR drug regimens (shorter and longer) are used [2, 13-15].

\section{Epidemiology}

FGTB is an important variety of EPTB which causes significant morbidity, especially menstrual dysfunction and infertility (both primary and secondary) in affected women [2-6]. The prevalence of FGTB in infertility patients varies from country to country being only $0.72 \%$ in Portugal, $1 \%$ in USA and Europe to 20\% in Pakistan and 6-25\% in India being more in tertiary referral centers and in assisted reproduction centers [16-20]. It also causes significant infertility in other south East Asian countries like Indonesia [21] FGTB is observed in younger age group (20-40 years) in South East Asia and Africa as compared to older (premenopausal) age group in Europe and USA possibly due to early child bearing in the developing countries $[2,5,6,10]$. Genital tuberculosis has significantly impacted both male and female reproduction in India [22, 23]. HIV coinfection has fueled the epidemic of TB all over the world with risk of TB in 10-20\% [24, 25]. Now, the COVID-19 pandemic has exerted deleterious effect on control of TB including EPTB and FGTB [26-28]. People ill with COVID-19 and pulmonary TB show similar symptoms such as cough, fever and difficult breathing, and both are transmitted through droplets infection. WHO has observed $25 \%$ global reduction in TB detection due to less attention to TB as all the resources went into control of COVID-19. It may be responsible for $26 \%$ increase in TB deaths in near future. WHO has advised all member nations to urgently maintain continuity of essential services for people affected with TB during the COVID-19 pandemic $[27,28]$.

WHO estimates that TB may have caused more than double the number of death of COVID-19 in 2020 but yet has not received much attention or funding. In fact, COVID-19, tuberculosis and poverty may be a potential destructive synergism causing a storm like situation [27, 28].

\section{Etiopathogenesis}

FGTB is caused by Mycobacterium tuberculosis but can also be rarely caused by Mycobacterium bovis due to consumption of unpasteurized milk in villages [2, 4]. Genital infection is usually secondary to pulmonary TB or intestinal TB through hematogenous or lymphatic route and is more common in immunocompromised people or with chronic medical diseases including HIV and COVID-19 [2, 5, 6, 24-28]. Among the various sites, fallopian tubes are mostly affected (90-100\%) followed by uterus $(70 \%)$, ovaries $(30 \%)$, cervix $(10 \%)$ and rarely vulva and vagina (1\% each) $[2,5,6$, 29]. Abdomino-pelvic TB may cause peritoneal adhesions, nodules and ascites and may simulate ovarian cancer [30]. Similarly, cervical TB may mimic cervical cancer causing abnormal vaginal discharge or vulval and vaginal ulcer or cancer [31].

Tuberculous of vulva may present as vulvul tumor or hypertrophic TB, while vaginal or vulval TB can present as genital fistula [2, 32].

In abdomino-pelvic $\mathrm{TB}$, we have observed various types of adhesions on laparoscopy as TB is notorious to cause thick and vascular adhesions which may make laparoscopy and laparotomy hazardous with more risk of injury to the bowel [4]. We have described perihepatic adhesions with hanging gall bladder sign in abdomino-pelvic TB [33]. We have also observed Sharma's ascending colon adhesion, Sharma's sigmoid colonic adhesive band, Sharma's compartmentalization sign in abdomino-pelvic TB [34-36]. We have described Sharma's parachute sign in abdomino-pelvic TB with ascending colon being adherent to anterior abdominal wall making putting second port of laparoscope risky and hazardous on right side [37]. Abdomino-pelvic TB may sometimes be coexistent with malignancies or endometriosis $[38,39]$.

\section{Symptomatology}

The clinical features of FGTB vary as per involvement of different genital organs and are shown in Table 1 [2-6]. It is important to know that up to $10-12 \%$ of women may be asymptomatic or may have no obvious signs on clinical examination. As in Table 1, commonest symptoms are menstrual dysfunction and infertility (both primary and secondary).

\section{Causes of Infertility}

1. Fallopian tubes are involved in almost all cases of FGTB. It is often bilateral causing exosalpingitis and endosalpingitis with tubal blockage. 
Table 1 Clinical feature of FGTB [2-5]

\begin{tabular}{|c|c|}
\hline Symptoms & Signs \\
\hline 1. No symptoms (10-12\%) & 1. No signs $(10-12 \%)$ \\
\hline 2. Constitutional symptoms (some time) & 2. Pallor \\
\hline \multicolumn{2}{|l|}{ (i) Fever } \\
\hline \multicolumn{2}{|l|}{ (ii) Weight loss } \\
\hline \multicolumn{2}{|l|}{ (iii) Anorexia } \\
\hline \multicolumn{2}{|l|}{ (iv) Malaise } \\
\hline \multicolumn{2}{|l|}{ (v) Night sweats } \\
\hline \multicolumn{2}{|l|}{ (vi) Feeling unwell } \\
\hline 3. Menstrual symptoms & 3. Swollen and inflammed bones with discharge (TB \\
\hline (i) Abnormal uterine bleeding (in early stages) & osteomyelitis) \\
\hline \multicolumn{2}{|l|}{ (ii) Postmenopausal bleeding } \\
\hline \multicolumn{2}{|l|}{ (iii) Oligomenorrhea } \\
\hline \multicolumn{2}{|l|}{ (iv) Hypomenorrhea } \\
\hline \multicolumn{2}{|l|}{ (v) Opsomenorrhea } \\
\hline \multicolumn{2}{|l|}{ (vi) Amenorrhea } \\
\hline \multicolumn{2}{|l|}{ (vii) Dysmenorrhea } \\
\hline 4. Infertility (common) & 4. Swollen and inflammed joints (skeletal TB) \\
\hline \multicolumn{2}{|l|}{ (i) Primary } \\
\hline \multicolumn{2}{|l|}{ (ii) Secondary } \\
\hline 5. Lower abdominal pain & 5 Lymphadenopathy (in lymph node TB) \\
\hline 6. Pelvic pain & 6. On chest auscultation (in pulmonary TB) Rales \\
\hline 7. Abdominal distension & 7. Abdominal distension and ascites (abdominal TB) \\
\hline 8 Abnormal vaginal discharge & 8. Bartholin enlargement vulval TB \\
\hline \multirow[t]{2}{*}{ 9. Symptoms of TB of other areas (as per site) } & 9. Swelling of vagina (Vaginal TB) \\
\hline & 10. Swelling or growth or ulcer on vulva (vulval TB) \\
\hline (A) Pulmonary TB & 11. Cervical growth or ulcer (cervical TB) \\
\hline (i) Cough & 12. Unhealthy vaginal discharge \\
\hline \multicolumn{2}{|l|}{ (ii) Sputum } \\
\hline \multicolumn{2}{|l|}{ (iii) Hemoptysis } \\
\hline \multicolumn{2}{|l|}{ (iv) Chest pain } \\
\hline (B) Tuberculous lymphadenitis of neck, axilla or groin & 13. Pus discharge through cervix (pyometra) \\
\hline \multicolumn{2}{|l|}{ Enlarged lymph nodes in different areas } \\
\hline (C) Skeletal TB & 14. Enlarged uterus (in pyometra) \\
\hline \multicolumn{2}{|l|}{ (i) Pain and swelling in joints } \\
\hline \multicolumn{2}{|l|}{ (ii) Pain, swelling and discharge from bones } \\
\hline (D) Intestinal TB & 15. Tubo-ovarian mass and tenderness \\
\hline (i) Constipation & (i) Unilateral \\
\hline (ii) Loose motion & (ii) Bilateral \\
\hline \multicolumn{2}{|l|}{ (iii) Abdominal distension } \\
\hline \multicolumn{2}{|l|}{ (iv) Abdominal swelling } \\
\hline \multirow{3}{*}{$\begin{array}{l}\text { 10. Acute abdominal pain due to rupture of abscess or flare-up of lesions usually after } \\
\text { some procedure }\end{array}$} & 16. Unusual features \\
\hline & (i) Vesicovaginal fistula \\
\hline & (ii) Rectovaginal fistula \\
\hline
\end{tabular}

2. Uterus: TB affects endometrial receptivity with damage of endometrium and formation of intrauterine synechiae (Asherman's syndrome). Latent FGTB can cause recurrent implantation failure and recurrent miscarriages due to endometrial hostility through increased TNF alpha (tumor necrosis factor alpha) and interleukin 2 (IL-2) levels [17]. It also shifts $\mathrm{T}$ helper cells response from $\mathrm{T} 1$ to $\mathrm{T} 2$. 
3. TB causes oophoritis with poor ovarian reserve and increased need of gonadotrophins for ovulation induction. It can also destroy ovaries [8, 9].

\section{Differential Diagnosis}

Abdomino-pelvic TB and FGTB are great mimickers and may be confused with other genital diseases like ovarian cyst, vulval or vaginal cyst, ectopic pregnancy, endometriosis genital malignancies and other intestinal diseases (appendicitis, Crohn's disease) actinomycosis and various miscellaneous conditions (Schistosomiasis, filariasis, silicosis, leprosy, granuloma inguinale). Hence, history taking, meticulous clinical examination and judicious investigations are required to make the appropriate diagnosis of FGTB and to rule out other diseases.

\section{Diagnosis of FGTB}

As FGTB can be asymptomatic and may mimic many other diseases, its diagnosis may be missed in many women, especially in early stages. Hence, a high index of suspicion is needed. A careful and detailed history of anti-tubercular past tuberculosis (especially pulmonary) including in childhood, family history of TB and past history of treatment, history of other chronic diseases or conditions causing poor immunity (including HIV, immunosuppressive treatment, diabetes) is crucial to make the diagnosis. A meticulous general and gynecological examination as per Table 2 is crucial to make the diagnosis of FGTB. Clinicians should be particularly vigilant to make the diagnosis of FGTB in non-sexually active women with tubo-ovarian masses (adnexal masses) after ruling out germ cell tumors. Similarly, chronic pelvic inflammatory disease or abdominal or pelvic pain not responding to routine treatment should also be suspected to have FGTB. A careful examination for TB at other sites can give an active diagnosis. We have observed discharging sinus on knee or cervical or axillary lymphadenopathy in adolescent girls with tubo-ovarian masses in FGTB patients. One can easily collect specimen from such sites for demonstration of acid fast bacilli as compared to poorly accessible

Table 2 Laboratory investigations and diagnostic modalities for FGTB [2-4, 11, 12, 29]

\section{S. no}

1. Hematological tests

Complete hemogram: anemia, total leucocyte count may be increased. Lymphocytosis and raised erythrocyte sedimentation rate may be present

2. Coinfection testing

i) Human immunodeficiency virus testing ii) $\mathrm{HbsAg}$ iii) $\mathrm{HCV}$

3. Blood sugar test

Blood sugar fasting and postprandial for diabetes mellitus

4. Other blood tests

Renal function tests: to rule out chronic kidney dresses

5

6.

7.

8.

9. Radiological tests

10. Endoscopic modalities
CA-125 levels on patient serum: A level of $>35$ IU/L may be seen in FGTB but is not reliable

Mantoux test: a value of $>10 \mathrm{~mm}$ may give some clue but is not reliable

Interferon gamma release assay (IGRA) has been approved by US FDA for diagnosis of latent TB and is available as Quantiferon TB-Gold which is done on whole blood

$\mathrm{X}$ ray chest for any active or old healed pulmonary $\mathrm{TB}$

Endometrial aspirate or biopsy or peritoneal or lesion biopsy It is the most important test. Various tests can be done on it [2-4]

(i) AFB microscopy

(ii) AFB culture

(iii) Cartridge-based nucleic acid amplification test (CBNAAT) or gene Xpert

(iv) PCR (polymerase chain reaction)

(v) Epithelioid granuloma on histopathology

(vi) Newer molecular tests (Xpert Ultra, TB-LAMP)

(i) Hysterosalpingography: It is avoided in acute disease to avoid flare-up. Various findings of FGTB can be blocked tubes, (usually cornual block) tobacco-pouch appearance of tubes, beaded tubes, filling defects in uterine cavity. (Asherman's syndrome)

(ii) Ultrasound: Cogwheel appearance due to hydrosalpinx is visible as tubal dilatation with septae due to tubal mucosal thickening. Endometritis due to TB causes thin, diffuse endometrial images with irregular borders

(iii) Computerized tomography (CT), magnetic resonance imaging (MRI) and positron emission tomography (PET): one of the three modalities can be used for tuberculous tubo-ovarian masses. MRI has higher resolution especially for TB lymphadenitis. PET-CT can demonstrate increased FDG uptake of tuberculous tubo-ovarian masses

(i) Diagnostic laparoscopy: best diagnostic tool to detect FGTB by direct visualization of TB lesions on tubes like tubercles and caseous nodules and to take biopsy

(ii) Hysteroscopy: to visualize endometrial disease like tubercles, pale endometrium and intrauterine adhesions 
adnexal masses, especially in non-sexually active women where endometrial biopsy cannot be done.

\section{Investigations}

Various detailed laboratory investigations and diagnostic modalities used in diagnosis of FGTB are shown with commonly used investigations being discussed here:

\section{Endometrial aspirate or sampling or peritoneal biopsy}

It is the most important investigation which should be done whenever possible especially in all sexually active women. It is done in premenstrual phase (day 21-24 of regular cycle) with patient having maintained abstinence (or used condoms) in the current cycle to avoid inadvertent pregnancy. It can be done with no 4 Karman cannula or pipelle or with endometrial biopsy curette. The sample should preferably be without blood and should be homogenized and sent in saline for following investigations.

(i) AFB microscopy using Ziehl-Neelsen (ZN) staining is a traditional, rapid and economic method to detect bacteria but lacks sensitivity [3, 29].

Auramine and rhodamine fluorescent dyes with LED microscopy improve sensitivity. Gabbett cold staining method is economic and fast [2, 3, 12, 29].

(ii) Mycobacterial culture: It is the gold standard for diagnosis of tuberculosis and is particularly useful for drug sensitivity testing with various media being discussed below [2, 3, 12, 29]

(a) Traditional egg-based solid media like Lowenstein Jensen (LJ) medium and agar-based 7H10 or $7 \mathrm{H} 11$ broth have been used for decades. They take up to 6 weeks to grow M. tuberculosis [2, 3, 12, 29]

(b) BACTEC 460 radiometric culture system: is not used in current practice due to risk of needle stick injury and radiation hazard.

(c) BACTEC-MGIT 960 system (Mycobacterium Growth Indicator Tube): It is a non-radio-active, noninvasive and fluorometric culture medium which uses Middlebrook $7 \mathrm{H} 9$ broth and fluorescent compound in a silicone sensor [2, 3, 12].

(d) Versa TREK: It is a US FDA-approved liquidbased culture for $\mathrm{M}$. tuberculosis with much higher detection rate of FGTB of up to $46.7 \%$ and uses special Myso bottle. Its advantages are higher specificity, sensitivity testing and for identification of Mycobacteria species. Limitations is longer time (weeks) for results with delay in treatment. (iii) Cartridge-based nucleic acid amplification test (CBNAAT): It is also called gene Xpert which uses heminested real-time PCR to magnify Mycobacterium tuberculosis. It can be chip-based (Truenat). It can detect as low as 133 colony-forming units (CFU)/ $\mathrm{ml}$ of specimen and is used to diagnose TB and can detect rifampicin resistance within $2 \mathrm{~h}$. It has been approved by WHO. We observed a sensitivity of $30 \%$ and specificity of almost $100 \%$ in FGTB patients with gene Xpert [29, 40, 41]. Xpert Ultra has much higher sensitivity. Disadvantages are lower sensitivity, higher cost, negative report due to blood contamination and inability to know resistance to other drugs.

(iv) Although PCR is a rapid, sensitive and specific tool to diagnose FGTB, it has many drawbacks. It cannot differentiate between live and dead bacteria and may remain positive during anti-tuberculous therapy or after completion of therapy. It thus has high false positivity and may be false negative also. Instead of uniplex PCR, multiplex PCR using multi-gene or multi-primer PCR has higher sensitivity. However, in clinical practice, PCR is not used to diagnose FGTB or to start anti-tubercular therapy but as a method to investigate further for FGTB using endoscopic techniques $[3,29,41]$.

(v) Loop-mediated isothermal amplification (LAMP): It is quicker and inexpensive with sensitivity of about $40 \%$ in detection of FGTB but cannot diagnose drug resistance [42]. Various studies are ongoing on it, and it is still not routine test for diagnosis of FGTB.

(vi) Molecular line probe assays (LPA): They have been recommended by WHO for rapid diagnosis of multidrug-resistant TB including FGTB on positive liquid culture and can detect drug sensitivity also.

(vii) Histopathology: A part of endometrial (or peritoneal) biopsy specimen is taken in $10 \%$ formalin for detection of epithelioid granuloma or granulomatous disease on histopathology with almost $100 \%$ specificity but very low sensitivity [29]. Typical caseation is rare in FGTB due to shedding of endometrium during menstruation.

\section{Newer Molecular Methods in Pipeline}

1. Newer generation sequencing (NSG) technology can allow increasingly rapid and accurate sequencing of entire Mycobacterial genome and has the potential for amplifying the diagnosis and management of drugsensitive and drug-resistant pulmonary and extra-pulmonary TB (including FGTB) but is not yet routinely available [43]. 
2. Nuclear Receptors: As nuclear receptors have important role in maintenance of uterine receptivity and immune modulation, they can be used as a biomarker for early detection of FGTB, especially latent FGTB and as a therapeutic drug target, but are still experimental [44].

\section{Laparoscopy}

It is a very valuable tool for infertility and FGTB with the dual advantage of pelvic organ visualization and sample collection even from inaccessible sites for laboratory diagnosis as biopsy can be taken under direct vision [2, 12, 45]. One should carefully visualize the pelvic organs (uterus, ovaries, tubes) and entire peritoneal cavity including upper abdomen for any TB lesions [3, 4]. We have observed various new signs perihepatic adhesions and Sharma's hanging gall bladder sign, Sharma's ascending colonic adhesion, Sharma's sigmoid colonic adhesive band and Sharma's parachute sign (adhesions of ascending colon to abdominal wall) and Sharma's compartmentalization sign (multiple compartments are formed by omental adhesions to contain infection) [33-37]. We have also observed fallopian tubes distended with alternate constrictions and dilatations on laparoscopy and dye test resembling blue python in FGTB cases (Sharma's blue python sign) [46] and fusion of both fallopian tubes due to FGTB (Sharma's kissing fallopian tubes sign) [47]. Figure 1 shows laparoscopy showing perihepatic adhesions (Fitz Hugh Curtis Syndrome) with Sharma's hanging gall bladder sign in a case of FGTB, while Fig. 2 shows laparoscopy showing Sharma's blue python sign with distended hydrosalpinx in left fallopian tube and tubercles on right fallopian tube and ovary in a case of FGTB.

\section{Hysteroscopy}

It is an endoscopic visualization of endometrial cavity using a distension medium (saline or glycine) and is often done concomitantly with laparoscopy for better results. The various TB lesions on hysteroscopy are pale endometrium, tubercles, caseous nodules, chronic endometritis, edema, micropolyps, varying grades of intrauterine adhesions and distorted and shrunken uterine cavity with obliterated ostia $[2,12,20]$. Figure 3 shows pale endometrium and intrauterine adhesions on hysteroscopy in a case of FGTB.

\section{Use of Composite reference standard (CRS) and diagnostic algorithm for FGTB (Fig. 4)}

In the absence of availability of any single sensitive and specific test for detection of FGTB, combination of tests can be used to increase the sensitivity and detection rate of FGTB and to avoid false positivity. High degree of clinical suspicion from the past or family history of TB or antituberculous therapy, menstrual dysfunction, especially oligomenorrhea and infertility along with combination of reliable tests, can detect higher number of FGTB cases as has been done for other extra-pulmonary TB (EPTB) cases like pleural TB [48]. Index TB guideline is an example of
Fig. 1 Laparoscopy showing perihepatic adhesions (FitzHugh-Curtis syndrome) (single arrow) with Sharma's hanging gall bladder sign (double arrow) in a case of FGTB

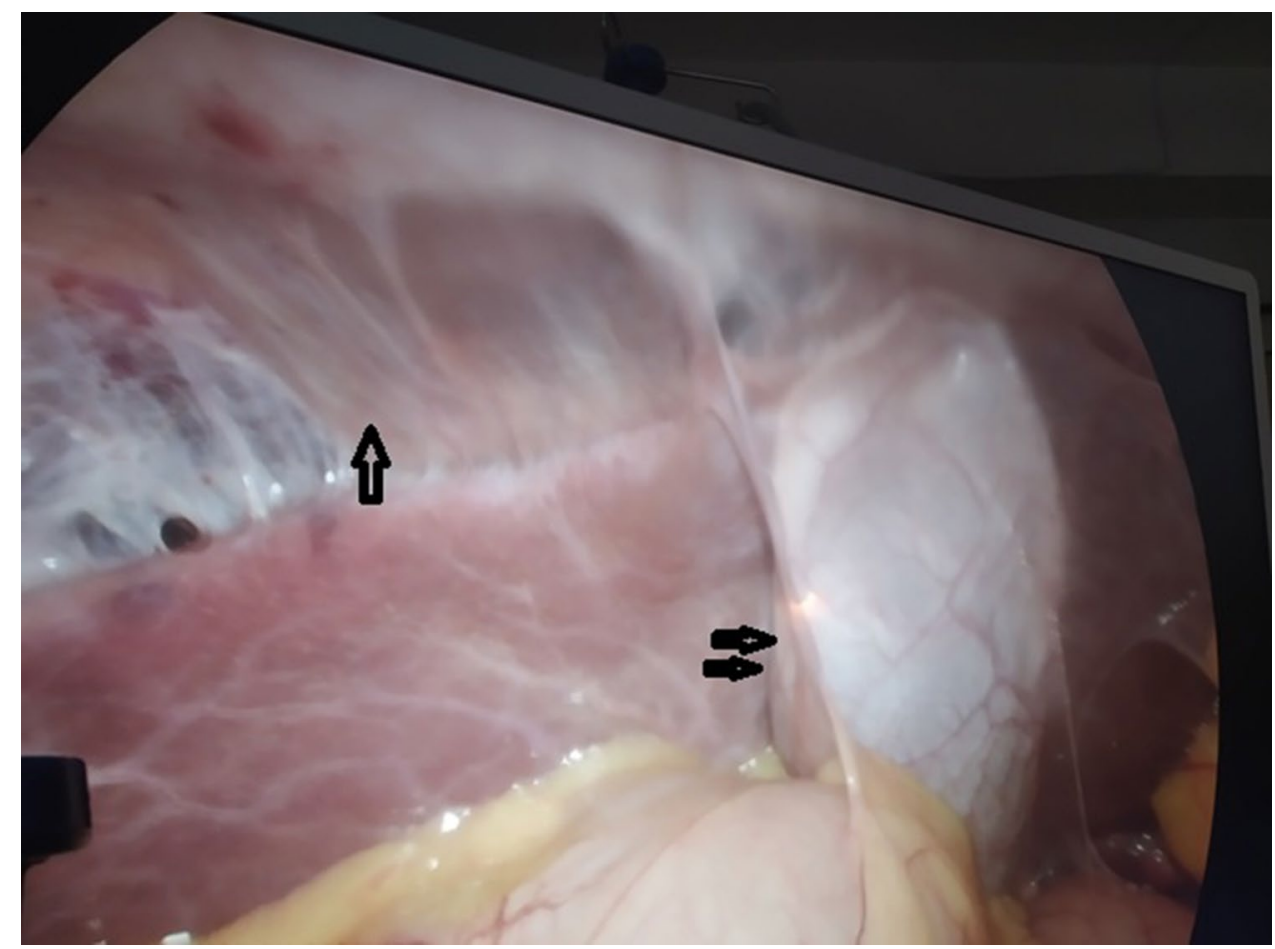


Fig. 2 Laparoscopy showing Sharma's blue python sign with distended hydrosalpinx in the left fallopian tube (single arrow) and tubercles (double arrow) on the right fallopian tube and ovary in a case of FGTB

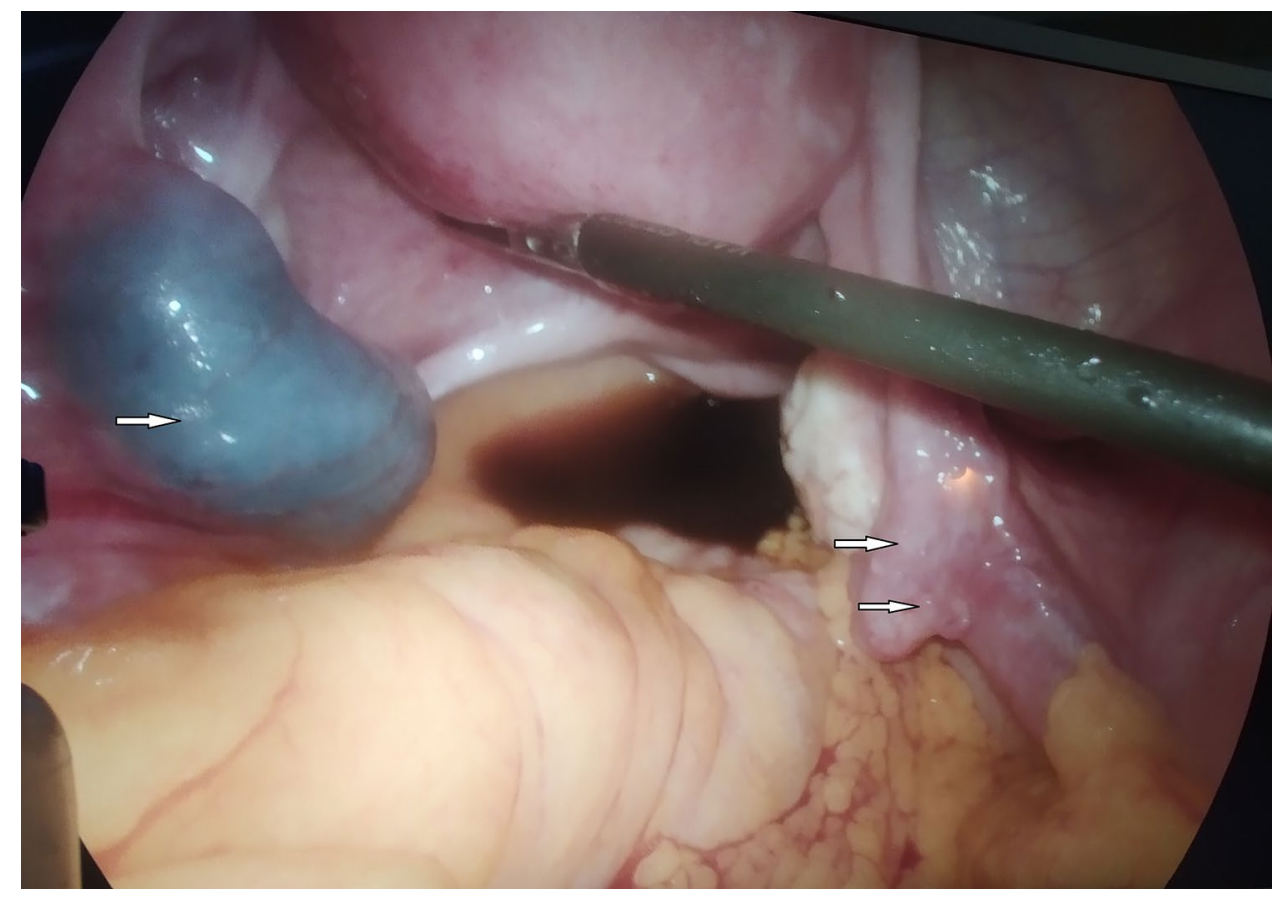

Fig. 3 Hysteroscopy showing pale endometrium and intrauterine adhesions in a case of FGTB

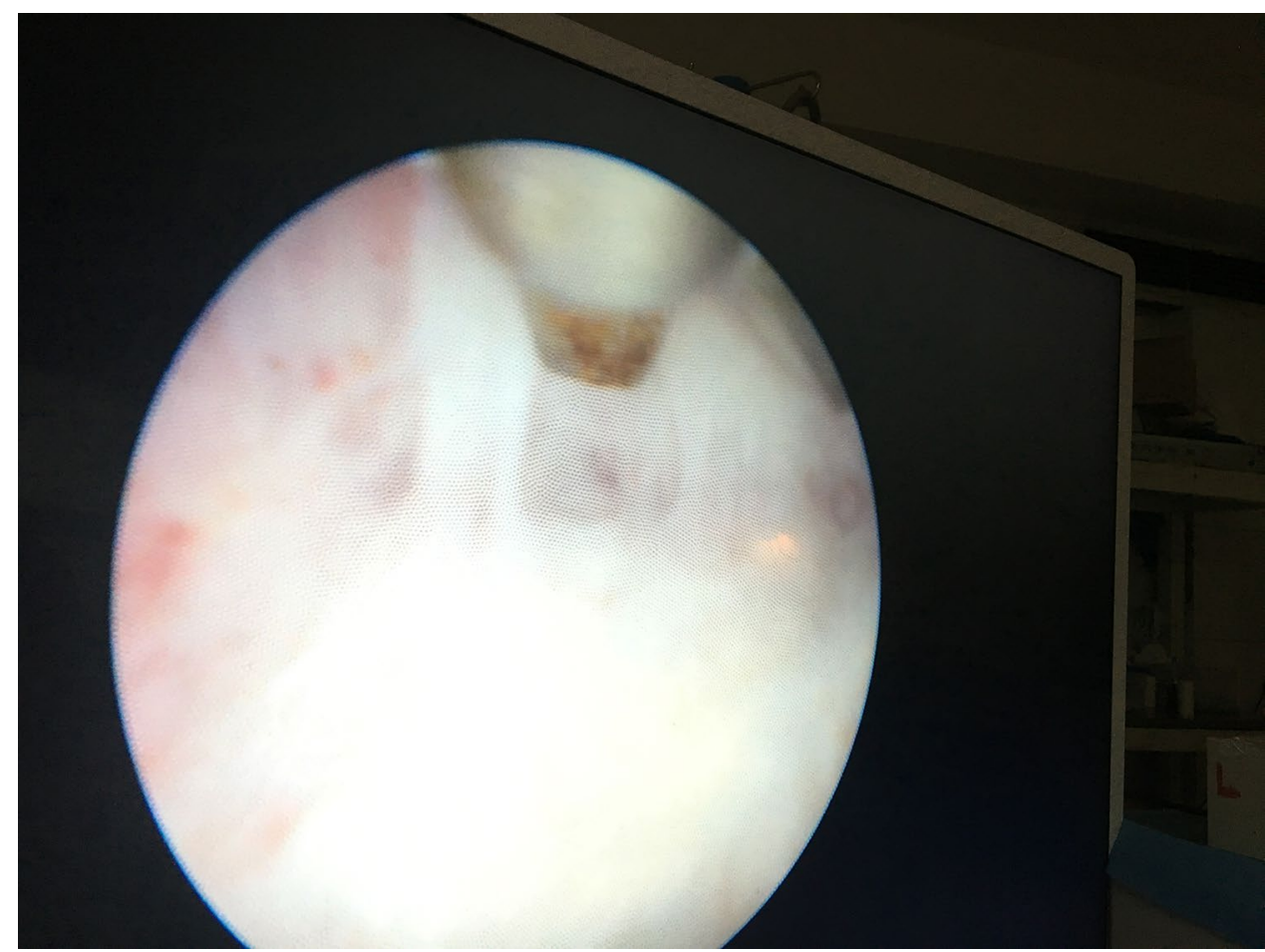

use of composite reference standard for various EPTB cases including FGTB [48]. It combines positive detection of acid fast bacilli on microscopy or culture, positive gene Xpert or positive epithelioid granuloma on histopathology of endometrial or peritoneal biopsy and definite findings of TB like tubercles, caseous nodules or beaded tubes on laparoscopy or hysteroscopy (tubercles, caseous nodules) [48].
An algorithm for diagnosis of FGTB is shown in Fig. 4. Thus, PCR alone is not used for diagnosis of FGTB.

\section{Medical treatment}

There have been changes in the treatment of both pulmonary and extra-pulmonary TB including FGTB, shown in 


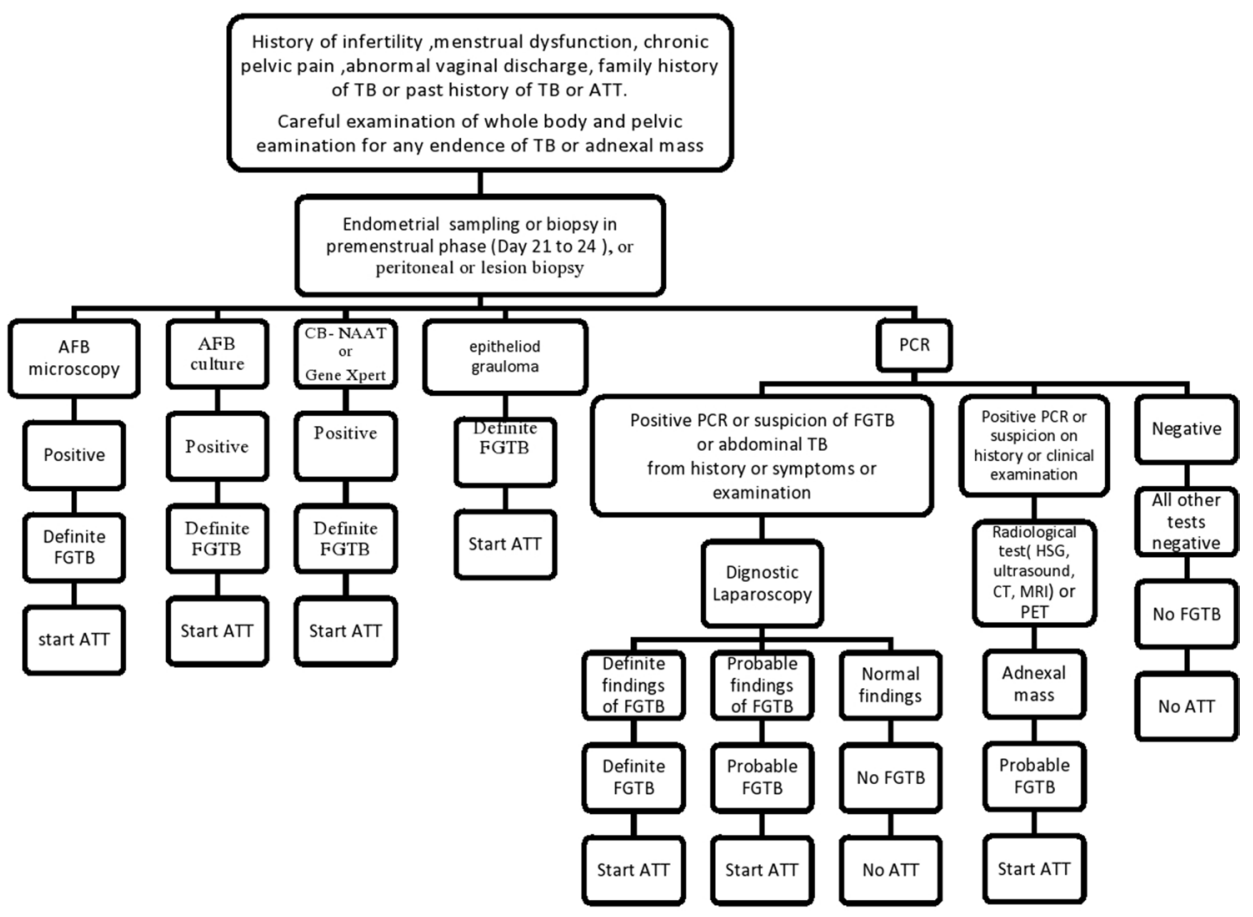

AFB : Acid-fast bacilli

ATT : Anti tuberculous therapy

FGTB : Female genital tuberculosis

CB NAAT: Cartridge based nucleic acid amplification test

PCR : Polymerase chain reaction
HSG: Hysterosalpingography

CT: Computed tomography

MRI : Magnetic resonance imaging

PET: Position emission tomography

Fig. 4 Use of composite reference standard (CRS) and diagnostic algorithm for FGTB

Table 3. Treatment of FGTB is similar to pulmonary TB and is given for total 6 months. It consists of four drugs regimen with rifampicin (r), isoniazid $(\mathrm{h})[1,2,13]$ pyrazinamide $(\mathrm{z})$ and ethambutol (E) daily orally for first 2 months of intensive phase $[1,2,13]$. In the continuation phase, three-drug regimen (unlike 2 drugs in past) is given using rifampicin (R), isoniazid (I) and ethambutol (E) orally daily for next 4 months [1, 2, 13] Categories are no longer used. Even patients with irregular treatment or defaulters are treated with the above regimen in the case of drug-sensitive TB.

For primary or secondary drug-resistant FGTB, longer oral regimen with reserve drugs is given for 18-20 months or shorter regimen is given for 9-12 months in selected category of patients as shown in Table 4 [2, 13, 14, 49]. All women should be carefully monitored for adherence to treatment and any side effects, especially hepatotoxicity with rifampicin, isoniazid and pyrazinamide, ocular toxicity (ethambutol) and peripheral neuritis (isoniazid). Pyridoxine can be given for any neuritis, and liver function tests should be performed on suspicion of hepatotoxicity.

Treatment should preferably be directly observed treatment short course (DOTS) with quality assured free medicines from government DOTS centers present all over India under National TB Elimination program (previously called Revised National TB Control Program).

\section{TB Notification}

India TB notification policy mandates that all patients with TB including FGTB are notified through Nikshay (TB notification portal).

Patients of TB including FGTB can also take benefit of government's Nikshay Poshan yojana in which TB patients gets Rs. 500 per months to buy food till patient continues treatment.

\section{Surgical Treatment}

Surgery is best avoided in FGTB due to more complications and need to give ATT. Only limited surgery in the form of drainage of abscesses can be done [2]. However, diagnostic laparoscopy and hysteroscopy is done at beginning to diagnose the disease and later after completion of full treatment of TB to prognosticate the patient and to plan further treatment for infertility. Treatment is planned as follows. 
Table 3 Drug treatment of drug-sensitive and isoniazid-resistant FGTB as per WHO and National TB Elimination Program (NTEP) (13-15)

Drug-sensitive FGTB (rifampicin- and isoniazid-sensitive new previously treated or retreatment TB cases)

\begin{tabular}{|c|c|c|c|}
\hline \multicolumn{4}{|c|}{ Daily dose regimen for adults as per weight bands } \\
\hline \multicolumn{4}{|c|}{ Number of tablets of fixed drug combination (FDC) } \\
\hline $\begin{array}{l}\text { Weight } \\
\text { category }\end{array}$ & $\begin{array}{l}\text { Intensive-phase } \\
\text { HRZE } 75 \mathrm{mg} \\
/ 150 \mathrm{mg} / 400 \mathrm{mg} / 275 \mathrm{mg} \\
\text { Per FDC tablet } \\
\text { ( } 2 \text { months) oral daily } \\
\text { treatment }\end{array}$ & $\begin{array}{l}\text { Continua- } \\
\text { tion-phase } \\
\text { HRE } 75 \mathrm{mg} \\
/ 150 \mathrm{mg} / 275 \mathrm{mg} \\
\text { per FDC tablet } \\
\text { (4 months) oral } \\
\text { daily treatment }\end{array}$ & $\begin{array}{l}\text { Strepto- } \\
\text { mycin* } \\
(\mathrm{g})\end{array}$ \\
\hline $25-39 \mathrm{~kg}$ & 2 & 2 & 0.5 \\
\hline $40-54 \mathrm{~kg}$ & 3 & 3 & 0.75 \\
\hline $55-69 \mathrm{~kg}$ & 4 & 4 & 1 \\
\hline$\geq 70 \mathrm{~kg}$ & 5 & 5 & 1 \\
\hline
\end{tabular}

*Streptomycin is given only for adverse drug reaction to first-line drugs like drug-induced hepatitis when HRZ are withheld and streptomycin, ethambutol and levofloxacin are given till liver function tests (LFT) return to normal, when RHZ are added sequentially under LFT monitoring

On an average a woman with $50 \mathrm{~kg}$ weight (45-54 $\mathrm{kg}$ ) will receive $225 \mathrm{mg}$ isoniazid $(\mathrm{H}), 450 \mathrm{mg}$ of rifampicin $\AA, 1200 \mathrm{mg}$ pyrazinamide $(\mathrm{Z})$ and $825 \mathrm{mg}$ ethambutol (E) while a $60-\mathrm{kg}$ woman (55-69) will receive $300 \mathrm{mg}(\mathrm{H}), 600 \mathrm{mg}{ }^{\circledR}, 1600 \mathrm{mg}(\mathrm{Z})$ and $1100 \mathrm{mg}(\mathrm{E})$, respectively

\section{Isoniazid-Resistant Rifampicin-Sensitive (Hr-TB)**}

REZ + levofloxacin: uniphasic orally daily for 6 months (LRZE for 6 months daily orally)

i. Regimen to be modified if polyresistance detected

ii. **Regimen extended to 9 months in the case of poor response, provided other resistance ruled out

iii. Levofloxacin to be replaced with moxifloxacin if strain resistant to levofloxacin but sensitive to moxifloxacin

1. If tubes are patent on laparoscopy and endometrium is normal on hysteroscopy, normal conception is tried or ovulation induction drugs are given.

2. If tubes are blocked, but endometrium is receptive (normal), IVF ET (in vitro fertilization and embryo transfer) is performed with good outcome and conception rate of up to $25 \%$.
3. If tubes are blocked and endometrium is not receptive with more than grade 3 adhesions (not amenable to surgical treatment), then gestational surrogacy is advised.

4. If tubes are blocked and endometrium is not receptive and ovaries are also damaged, then adoption is advised.

\section{TB and Pregnancy}

Both pulmonary and extra-pulmonary TB can occur in pregnancy. Drug-sensitive TB must be treated with all the four drugs (RHZE) for 2 months and the three drugs (RHE) for 4 months even in first trimester of pregnancy [2]. Drugresistant TB patients should not conceive while on reserve drugs. Medical termination of pregnancy (MTP) is advised for MDR TB in first trimester as reserve drugs can be teratogenic. If patient refuses MTP, then treatment is modified by excluding injections (kanamycin, amikacin, gentamycin, as they are fetotoxic) avoiding ethionamide till 32 weeks and after counseling the patient. Use of bedaquiline though safe is still debatable in pregnancy.

\section{Summary and Conclusions}

FGTB is a significant factor to cause reproductive and genital morbidity, especially infertility in the developing nations. The clinicians should always keep FGTB in mind and should take detailed history and careful examination and perform appropriates investigations to reach to a diagnosis of FGTB. Use of composite reference standard increases detection rate. Treatment of drug sensitivity FGTB is essentially medical using four drugs (RHZE) daily orally for 2 months followed by three drugs (RHE) daily orally for next 4 months (total 6 months). For drug-resistant FGTB, all oral longer regimens (18-20 months) or shorter regimen (9-12 months) are used under careful supervision. In vitro fertilization and embryo transfer and surrogacy have a role in selected cases. Newer molecule tests like genomics and nucleus receptors are being developed for early diagnosis of TB including FGTB [50]. Novel biotechniques, nanoparticles and stem cell transplantation are being developed for regeneration of endometrium (in Asher man's syndrome), fallopian tubes (fallopian tubes blockage and damage) and for ovaries (damaged ovaries). 
Table 4 Drug treatment of drug-resistant FGTB as per WHO and National TB Elimination Program (NTEP) (13-15)

(A) Longer all oral regimen (18-20 months)

Indications: (II MDR FGTB):

1. Disseminated RR/MDR FGTB with pulmonary or other extra-pulmonary TB sites/involvements

2. RR/MDR with resistance to other reserve drugs

3. History of intake of reserve drugs for $\geq$ one month

4. Intolerance or adverse drug reactions on shorter MDR regimen

5. HIV-positive patients with RR/MDR FGTB

6. Pregnant women with MDR TB. It can be given to all patients of MDR

S No
Name of drug

Tab bedaquiline
Tab levofloxacin or Tab moxifloxacin

Tab linezolid

Tab cycloserine

Tab clofazimine

Tab ethionamide*
Dose

$400 \mathrm{mg}$ daily for 2 weeks then $200 \mathrm{mg}$ thrice weekly for 22 weeks ( total 24 weeks)

$750 \mathrm{mg}$ or $600 \mathrm{mg}$

$600 \mathrm{mg}$

$500-750 \mathrm{mg}$

$100 \mathrm{mg}$

$750 \mathrm{mg}$

These 6 drugs are given orally daily for 6-8 months in intensive phase

In continuation phase, 4 drugs: Tab levofloxacin /moxifloxacin, linezolid, cycloserine and clofazimine are given for 12 months for MDR TB and 14 months for XDR TB.

(Resistance to fluoroquinolones with or without resistance to other group A drugs)

Linezolid can be given on alternate days during continuation phase

*Ethionamide in some cases (not all cases) Replacement sequence in case of drug intolerance is as follows: delamanid, aminoglycoside, pyrazinamide, ethionamide, pas, ethambutol, carbapenem

(B) Shorter drug-resistant TB regimen (total 9-12 months regimen) (7 drugs for 4-6 months followed by 4 drugs for 5 months) (Total 9-11 months)

Indications:

1 Localized RR/ MDR FGTB

2. History of intake of reserve drugs $\leq$ one month

3. Sensitivity to fluoroquinolones, second line injectables, $\mathrm{Z}$ (inhA or kat $\mathrm{G}$ mutation but not both)

Intensive phase (4-6 months Am-Mfx-Cfz-Eto-High H-Z-E)

\begin{tabular}{|c|c|c|}
\hline 1 & Injection amikacin\# $(\mathrm{Km})$ (intramuscular) & $750 \mathrm{mg}$ \\
\hline 2 & Tab moxifloxacin (Mfx) & $600 \mathrm{mg}$ \\
\hline 3 & Tab clofazimine ( Cfz) & $100 \mathrm{mg}$ \\
\hline 4 & Tab ethionamide (Eto) & $750 \mathrm{mg}$ \\
\hline 5 & High dose isoniazid (high $\mathrm{H}$ ) & $1000 \mathrm{mg}$ \\
\hline 6 & Tab pyrazinamide $(\mathrm{Z})$ & $1500 \mathrm{mg}$ \\
\hline 7 & Tab Ethambutol (E) & $1200 \mathrm{mg}$ \\
\hline \multicolumn{3}{|c|}{ Continuation phase ( 5 months of daily oral Mfx-Cfz-Z-E) } \\
\hline 1 & Tab moxifloxacin (Mfx) & $600 \mathrm{mg}$ \\
\hline 2 & Tab clofazimine $(\mathrm{Cfz})$ & $100 \mathrm{mg}$ \\
\hline 3 & Tab pyrazinamide $(\mathrm{Z})$ & $1500 \mathrm{mg}$ \\
\hline 4 & Tab ethambutol (E) & $1200 \mathrm{mg}$ \\
\hline
\end{tabular}

Pyridoxine $40 \mathrm{mg}$ is given daily for prophylaxis (100 mg for treatment) for adverse drug effects in all cases for all drug-sensitive and drugresistant regimens. \# Bedaquiline replaced shorter all oral regimen is being pilot-tested for RR and MDR TB with replacement of amikacin with bedaquiline with total duration of 9-12 months in eligible patients

Author contributions SJB contributed to the conceptual the idea, project development and manuscript writing. SE contributed to manuscript writing and taking endometrial samples. SS contributed to manuscript editing and proof reading. DS contributed to data collection.

Conflict of interest The authors declare that they have no conflict of interest.

\section{References}

1. World Health Organization. Global Tuberculosis Report-2019. https://apps.who.int/iris/bitstream/handle/10665/329368/97892 41565714-eng.pdf? Accessed 1 Aug 2020.

2. Sharma JB, Sharma E, Sharma S, et al. Genital tb-diagnostic algorithm and treatment. Indian J Tuberc. 2020;67(4S):S111-8. 
3. Munne KR, Tandon D, Chauhan SL, et al. Female genital tuberculosis in light of newer laboratory tests: a narrative review. Indian J Tuberc. 2020;67(1):112-20.

4. Sharma JB, Sharma E, Sharma S, et al. Female genital tuberculosis: revisited. Indian J Med Res. 2018;148(Supp):S71-83.

5. Grace A, Devaleenal B, Natarajan M. Genital tuberculosis in females. Indian J Med Res. 2017;145(4):425-36.

6. Sharma JB. Current diagnosis and management of female genital tuberculosis. J Obstet Gynaecol India. 2015;65:362-71.

7. Rajaram S, Gupta P, Gupta B, et al. Laparoscopy in the diagnosis of tuberculosis in chronic pelvic pain. Int J Mycobacteriol. 2016;5:318e323.

8. Tal R, Simoni M, Pal L. Latent and genital tuberculosis in the infertile population in US-experience at an academic fertility center in the north east underscores a need for vigilance. Fertil Steril. 2017;108:117e118.

9. Tal R, Lawal T, Granger E, et al. Genital tuberculosis screening at an academic fertility center in the United States. 2020.

10. Reis-de-Carvalho C, Monteiro J, Calhaz-Jorge C. Genital tuberculosis role in female infertility in Portugal. Arch Gynecol Obstet. 2021;6:66.

11. Mahajan N, Naidu P, Kaur SD. Insight into the diagnosis and management of subclinical genital tuberculosis in women with infertility. J Hum Reprod Sci. 2016;9(3):135-44.

12. Malhotra N, Singh UB, Iyer V, et al. Role of laparoscopy in the diagnosis of genital TB in infertile females in the era of molecular tests. J Minim Invasive Gynecol. 2020;27(7):1538-44.

13. Central TB Division of Ministry of Health and Family Welfare under Government of India. National Strategic Plan for Tuberculosis: 2017-2025, Elimination by 2025 [Internet]. 2017. https:// tbcindia.gov.in/WriteReadData/NationalStrategicPlan2017-25. pdf. Accessed 3 Aug 2020.

14. World Health Organization Guidelines for treatment of drugsusceptible tuberculosis and patient care. Treatment Tuberculosis World Health Organisation, geneva 2017 Update.

15. World Health Organization. WHO consolidated guidelines on tuberculosis: module 4: treatment: drug-resistant tuberculosis treatment. World Health Organization; 2020. https://apps.who. int/iris/handle/10665/332397. Accessed 1 Aug 2020.

16. Langer AJ, Navin TR, Winston CA, et al. Epidemiology of tuberculosis in the United States. Clin Chest Med. 2019;40(4):693-702.

17. Zahoor D, Bhat MM, Kanth F, et al. Prevalence of genital tuberculosis in infertile women; a study from a Tertiary Care Center in North India. IJCMR. 2019;6(6):F1-3.

18. Agarwal S, Chauhan J, Vachhani A, et al. A study on co-relation of infertility and female genital tuberculosis. Natl J Med Res. 2016;6(2):151-4.

19. Sharma D, Depan A, Yadav K, et al. Prevalence of female genital tract tuberculosis in suspected cases attending Gynecology OPD at tertiary centre by various diagnostic methods and comparative analysis. Int J Reprod Contracept Obstet Gynecol. 2019;8(6):2286-92.

20. Sharma JB, Dharmendra S, Agarwal S, et al. Genital tuberculosis and infertility. Fertil Sci Res. 2016;3:6-18.

21. Djuwantono T, Permadi W, Septiani L, et al. Female genital tuberculosis and infertility: serial cases report in Bandung, Indonesia and literature review. BMC Res Notes. 2017;10(1):683.

22. Malik S. Genital tuberculosis and its impact on male and female infertility. US Endocrinol. 2020;16(2):97-103.
23. Symptom Export and Treatment www.webmed.com World Health Organization Tuberculosis and HIV. Who Geneva 2015.

24. Bruchfeld J, Correia-Neves M, Källenius G. Tuberculosis and HIV coinfection. Cold Spring Harb Perspect Med. 2015;5(7):a017871.

25. Oberoi S, Khaira R, Rai SK. Aetiology of infertility: an epidemiological study. Nat J of Comm Med. 2017;8(1):17-20.

26. WHO information note. Tuberculosis and COVID-19 www.WHO. Int. Accessed 15 Dec 2020.

27. Saunders MJ, Evans CA. COVID-19, tuberculosis and poverty: preventing a perfect storm. Eur Respir J. 2020;56(1):2001348.

28. Hogan AB, Jewell BL, Sherrard-Smith E, et al. Potential impact of the COVID-19 pandemic on HIV, tuberculosis, and malaria in low-income and middle-income countries: a modelling study. Lancet Glob Health. 2020;8(9):e1132-41.

29. Meenu S, Ramalingam S, Sairam T, et al. Comparison of polymerase chain reaction (PCR), microbiological and histopathological observations in the diagnosis of endometrial tuberculosis. J Obstet Gynaecol India. 2020;70(6):510-5.

30. Cruz RP, Duarte M da R, Bianchi AR, et al. Pelvic tuberculosis masses mimicking advanced ovarian cancer: case report and literature review. J Gynecol Surg. 2020. https://doi.org/10.1089/gyn. 2020.0082.

31. Kumari R, Vaishya V, Khanna G, et al. Tuberculosis of the cervix: an uncommon cause of vaginal discharge in a post-menopausal woman. Nath Med J. 2018;31:140-50.

32. Sharma JB, Gupta S, Kriplani A, et al. Hypertrophic tuberculosis vulva an unusual case report. J Ind Med Assoc. 2021;119(2):41-2.

33. Sharma JB. Sharma's hanging gall bladder sign: a new sign for abdomino-pelvic tuberculosis: AN observational study. IVF Lite. 2015;2:94-8.

34. Sharma JB. Sharma's ascending colonic adhesion: a new sign in abdomino-pelvic tuberculosis with infertility. IVF Lite. 2016;3:18-22.

35. Sharma JB. Sharma's sigmoid colonic adhesive band-a new laparoscopic sign in female genital tuberculosis. Indian J Tuberc. 2020;67(3):327-32.

36. Sharma JB. Sharma's abdominal compartmentalization sign: a new laparoscopic sign for abdomino-pelvic tuberculosis. Indian J Tuberc. 2020;67(4):578-85.

37. Sharma JB. Sharma's parachute sign a new laparoscopic sign in abdomino pelvic tuberculosis. Indian J Tubercul. 2019. https:// doi.org/10.1016/j.ijtb.2019.06.004.

38. Gupta A, Gupta S, Manaktala U, et al. Coexisting genital malignancies with tuberculosis: a case series with review of literature. J Midlife Health. 2016;7(4):159-62.

39. Sharma JB, Goyal M, Kumar S, et al. Concomitant female genital tuberculosis and endometriosis. Indian J Tuberculosis. 2017;64(3):173-7.

40. Sharma JB, Dharmendra S, Jain S, et al. Evaluation of Gene Xpert as compared to conventional methods in diagnosis of female genital tuberculosis. Eur J Obstet Gynecol Reprod Biol. 2020;255:247-52.

41. Chaudhary R, Dhama V, Singh M, et al. Comparison of diagnostic accuracy of bactec culture, gene-xpert and histopathology in the diagnosis of genital tuberculosis in women with infertility. Int J Reprod Contracept Obstet Gynecol. 2020;9(4):1614-21.

42. Sethi S, Dhaliwal L, Dey P, et al. Loop-mediated isothermal amplification assay for detection of Mycobacterium 
tuberculosis complex in infertile women. Indian J Med Microbiol. 2016;34(3):322-7.

43. Jeanes C, O'Grady J. Diagnosing tuberculosis in the 21 st century-dawn of a genomics revolution? Int J Mycobacteriol. 2016;5(4):384-91.

44. Gupta S, Gupta P. Etiopathogenesis, challenges and remedies associated with female genital tuberculosis: potential role of nuclear receptors. Front Immunol. 2020;11:02161.

45. Mala YM, Prasad R, Singh N, et al. Role of laparoscopy in diagnosing genital tuberculosis in suspected women: a cross-sectional study from a tertiary care hospital in Northern India. Indian J Tuberc. 2018;65(1):23-9.

46. Sharma JB. Sharma's python sign: a new tubal sign in female genital tuberculosis. J Lab Phys. 2016;8(2):120-2.

47. Sharma JB. Sharma's kissing fallopian tubes sign: a new tubal sign in female genital tuberculosis. J Obstet Gynaecol India. 2017;67(3):227-9.

48. Ministry of Health and Family Welfare, Government of India and World Health Organization. INDEX TB Guidelines: guidelines on extra-pulmonary tuberculosis for India. New Delhi: MOHFW; 2016.

49. Sharma JB, Kriplani A, Sharma E, et al. Multi drug resistant female genital tuberculosis: a preliminary report. Eur J Obstet Gynecol Reprod Biol. 2017;210:108-15.

50. Korma W, Mihret A, Chang Y, et al. Antigen-specific cytokine and chemokine gene expression for diagnosing latent and active tuberculosis. Diagnostics. 2020;10(9):716.

Publisher's Note Springer Nature remains neutral with regard to jurisdictional claims in published maps and institutional affiliations.

\section{About the Author}

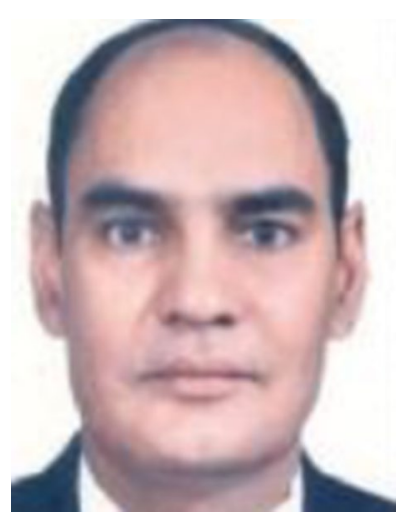

Dr. J. B. Sharma MD, DNB, FRCOG (London), PhD, MFFP FAMS, FICOG FIMSA, is a Professor in Department of Obstetrics and Gynecology, All India Institute of Medical Sciences, New Delhi. Before that he has worked as Professor in Maulana Azad Medical College, New Delhi. He is the recipient of the Dr. B C Roy award for Research in 2015. He is the Chairperson of the FOGSI Urogynaecology society (2020-2022). He has over 400 publications and has 250 peer reviewed articles in various journals of national and international repute. He is currently Editor in chief of Indian Obstetrics and Gynecology, Journal of Paediatrics, Obstetrics and Gynecology (JPOG) and Associate Editor of International Journal of Gynecology and Obstetrics, India. He has edited three books and has been awarded many times by Royal College of Obstetricians and Gynaecologists (RCOG), London. His special areas of interest include female genital tuberculosis, urogynecology and anemia in pregnancy. 